\title{
Molecular dynamics simulation of polymerlike thin films irradiated by fast ions: A comparison between FENE and Lennard-Jones potentials
}

\author{
N. W. Lima, ${ }^{1}$ L. I. Gutierres, ${ }^{1}$ R. I. Gonzalez, ${ }^{2}$ S. Müller, ${ }^{1}$ R. S. Thomaz, ${ }^{1}$ E. M. Bringa, ${ }^{3}$ and R. M. Papaléo ${ }^{1}$ \\ ${ }^{1}$ Faculdade de Física Pontifícia Universidade Católica do Rio Grande do Sul, Avenida Ipiranga, 6681, 90619-900 Porto Alegre, Brazil \\ ${ }^{2}$ Departamento de Física, Facultad de Ciencias, CEDENNA, Universidade de Chile, Casilla 653, \\ Santiago 78000024, Chile \\ ${ }^{3}$ CONICET and Facultad de Ciencias Exactas y Naturales Universidad Nacional de Cuyo, Mendoza, Argentina
}

(Received 5 August 2016; published 11 November 2016)

\begin{abstract}
In this paper, the surface effects of individual heavy ions impacting thin polymerlike films were investigated, using molecular dynamics simulations with the finite extensible nonlinear elastic (FENE) potential to describe the molecular chains. The perturbation introduced by the ions in the lattice was modeled assuming that the initial excitation energy in the ion track is converted into an effective temperature, as in a thermal spike. The track was heated only within the film thickness $h$, leaving a nonexcited substrate below. The effect of decreasing thickness on cratering and sputtering was evaluated. The results were compared to experimental data of thin polymer films bombarded by $\mathrm{MeV}-\mathrm{GeV}$ ions and to simulations performed with the Lennard-Jones potential. While several qualitative results observed in the experiments were also seen in the simulations, irrespective of the potential used, there are important differences observed on FENE films. Crater dimensions, rim volume, and sputtering yields are substantially reduced, and a threshold thickness for molecular ejection appears in FENE simulations. This is attributed to the additional restrictions on mass transport out of the excited track region imposed by interchain interactions (entanglements) and by the low mobility of the molten phase induced by the spike.
\end{abstract}

DOI: 10.1103/PhysRevB.94.195417

\section{INTRODUCTION}

High-energy charged particles have been widely used to characterize and modify materials in a controlled way, leading to important applications in different fields of materials science and engineering, medicine, and geosciences [1-4]. Sufficiently fast ions deposit a large amount of localized electronic excitation along its path through the material, which can be coupled to the lattice [5] and produce radiation damage in the bulk [2], as well as sputtering and mass transport at the near surface [6]. In particular, for polymers and other organic materials, the radiation effects of swift heavy ions are very pronounced, involving complex and irreversible chemical rearrangements along the ion path and large yields of particle emission. Sputtering in the electronic stopping regime is very efficient for organic materials [7], and large craters are often found at the point of ion incidence $[6,8]$.

The use of electronic sputtering for characterizing organic materials has gained special attention in recent years $[9,10]$. This stems from the fact that secondary ion mass spectrometry with $\mathrm{MeV}$ ions (MeV-SIMS) shows higher sputtering yields and less molecular fragmentation, when compared to keV-SIMS, and it is capable of molecular mapping of samples, including of tissues at high pressure atmospheres [10-12]. Even though several basic aspects of electronic sputtering have already been clarified since the early works in the field [7,13], many mechanistic details, important for mass spectrometry applications, are still not well understood [14]. One example is the complex and transient processes of energy deposition and transport leading to sputtering, which are not easily accessed via experimental observations. In this respect, there are important gaps that only computer simulations can provide adequate inputs. This includes details of fast (femtosecond) energy transport by secondary electrons using Monte Carlo (MC) simulations [15], or tracking the subsequent dynamics (on a nanosecond time scale) of the atoms set in motion by molecular dynamics (MD) simulations [16-26].

To simulate nonequilibrium energy transport and sputtering from ion tracks in molecular solids, the simplest approach is to employ the Lennard-Jones (LJ) potential, suited to model van der Waals solids such as condensed gases $[17,24,25]$. LJ has been also applied to explain several features of the electronic sputtering of biomolecules in the pioneering work of Fenyö et al. [26], and later to describe cratering in polymers as a function of the excitation density in the tracks [19]. More recently, simulations with LJ have also been used to evaluate the effect of spatial confinement of ion tracks on the cratering and sputtering of ultrathin polymer films irradiated by swift ions, with good qualitative agreement with experimental observations [27]. Despite the low computational cost associated with the Lennard-Jones potential, there are limitations on the simulation predictions, which stem from oversimplifications of the model.

In this paper, we extend our previous simulations of surface effects caused by the impact of individual swift heavy ions on LJ thin films [27], by explicitly including a molecular chain. We chose to simulate a polymer chain at the coarse-grained level, in which each monomer is represented as a particle bound to its neighbors via a finite extensible nonlinear elastic (FENE) potential [28]. The FENE potential has been successfully used to investigate the viscoelastic and rheological properties of polymers and their dependence on chain length [29,30]. Other coarse-grained models have been already used to study the effects of energetic ions in polymers, but only at the lowenergy regime [31,32], where nuclear collisions dominate. In our simulations using the FENE potential, craters, sputtering yields, and flow of molten material are substantially reduced, as compared to the $\mathrm{LJ}$ system. 
TABLE I. Parameters used for LJ and FENE potentials.

\begin{tabular}{lcc}
\hline \hline \multirow{2}{*}{ Parameter } & \multicolumn{2}{c}{ Value } \\
\cline { 2 - 3 } & Physical units & LJ reduced units \\
\hline$\sigma$ & $0.5 \mathrm{~nm}$ & 1 \\
$\epsilon$ & $0.07 \mathrm{eV}$ & 1 \\
$\tau_{0}$ & $1.7 \mathrm{ps}$ & 1 \\
$R_{T}$ & $3 \mathrm{~nm}$ & 6 \\
$K$ & $1.3 \mathrm{~N} / \mathrm{m}$ & 30 \\
$R_{0}$ & $0.75 \mathrm{~nm}$ & 1.5 \\
\hline \hline
\end{tabular}

\section{MD SIMULATIONS}

Classical molecular dynamics simulations were performed using the large-scale atomic/molecular massively parallel simulator (LAMMPS) [33] with conditions similar to what has been applied in previous simulations of cratering in polymers [19,27]. Polymer molecules were modeled as a chain of monomers with intrachain bonds governed by the FENE potential and interchain interactions modeled by the LJ potential. Monomers are assumed to be structureless rigid bodies. Lennard-Jones and FENE potentials are given in Eqs. (1) and (2), respectively:

$$
\begin{aligned}
E_{\mathrm{LJ}}= & 4 \epsilon\left[\left(\frac{\sigma}{r}\right)^{12}-\left(\frac{\sigma}{r}\right)^{6}\right], \\
E_{\mathrm{FENE}}= & -0.5 K R_{0}^{2} \ln \left[1-\left(\frac{r}{R_{0}}\right)^{2}\right] \\
& +4 \epsilon\left[\left(\frac{\sigma}{r}\right)^{12}-\left(\frac{\sigma}{r}\right)^{6}\right]+\epsilon .
\end{aligned}
$$

In both equations, $\sigma$ has space units, $\epsilon$ has energy units, and $r$ is the interatomic distance. In the FENE potential, $R_{0}$ indicates the maximum distance allowed between monomers directly bonded in the same chain and $K$ has energy/space ${ }^{2}$ units and it corresponds to the elastic constant of the system for small interatomic distances. The parameters used for both potentials are described in Table I. Note that in the FENE construction, as $r \rightarrow R_{0}, E_{\mathrm{FENE}} \rightarrow \infty$, which hinders adjacent particles in a chain to move farther than $R_{0}$. This means that radiolytic processes such as chain scission, production of small fragments, and bond rearrangements observed in real organic materials are not allowed in our simulations. Thus, the LJ and FENE approaches correspond to two extreme cases of weakly and very strongly bonded chains.

The simulation boxes contained about $5 \times 10^{6}$ particles within a volume of $80 \times 80 \times 80 \mathrm{~nm}^{3}$, including particles of the film and substrate. The boxes were centered at the origin and had a top free surface perpendicular to the $z$ axis. All other surfaces included a region of Langevin thermostating and damping with a thickness of $2 \sigma$. Periodic boundary conditions were used in the $x$ and $y$ directions (the spatial coordinates are defined in Fig. 1). The interchain potential parameters, $\sigma=0.5 \mathrm{~nm}$ and $\epsilon=0.07 \mathrm{eV}$, followed those values previously used to simulate cratering on pure LJ films [19,27]. Chains containing 100 monomers were

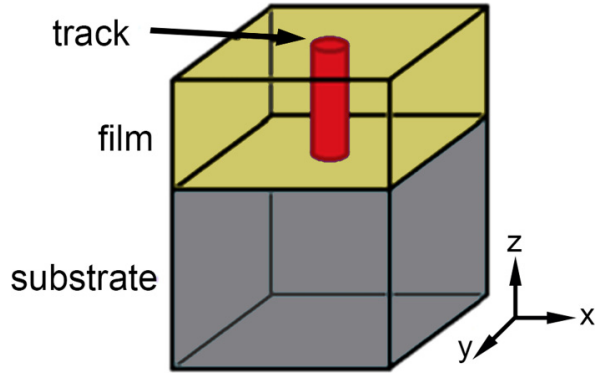

FIG. 1. Scheme of the simulation box showing the film, substrate, and the ion track. The ion track (red) is parallel to the $z$ axis and normal to the surface, and has a length equal to the film thickness.

used, with each monomer represented as a particle with a mass of $1.66 \times 10^{-22} \mathrm{~g}(100 \mathrm{u})$. This corresponds to a chain of poly(methyl methacrylate) (PMMA) with $M_{w}=10000 \mathrm{u}$. Figure 2 shows the position of 50 molecules in the beginning of a simulation, where it is possible to see that several chains are entangled. Entanglements provide additional constraints to molecular motion, as in a real polymer. The mean radius of gyration was about $2 \mathrm{~nm}$. The amorphous LJ samples were obtained following the methodology described in Ref. [18]. The noncrystallinity of both FENE and LJ samples was verified by evaluating the radial distribution function. The surfaces of the LJ samples were almost atomically flat, but FENE samples had a natural roughness, with root mean square values around $0.25 \mathrm{~nm}$ [Figs. 2(b) and 2(c)], close to the values found for polymer thin films on Si substrates by atomic force microscopy.

In order to describe the energy deposition by the ion, a thermal spike model was used, as presented previously [19,27]. Particles within a radius of $3 \mathrm{~nm}$ from the impact point were heated (in about $0.4 \mathrm{ps}$ ) by giving them energies corresponding to a temperature of $25000 \mathrm{~K}$. This forms a region equivalent to the ion track core. The simulations then followed the evolution of the system for about 400 ps, when crater features were already stabilized. The amount of energy deposited in the system per unit length (i.e., the effective stopping power) was evaluated by computing the difference in mechanical energy (kinetic plus potential) of the track atoms before and immediately after the heating process was completed, divided by the track length. The effective stopping power $(d E / d x)_{\text {eff }}$ gives the portion of the total electronic $d E / d x$ that is converted into atomic motion (usually assumed to be around $10 \%-40 \%)[18,34]$. The $(d E / d x)_{\text {eff }}$ associated with an initial track temperature of $25000 \mathrm{~K}$ is $\sim 1 \mathrm{keV} / \mathrm{nm}$. This was the value used in previous simulations with Lennard-Jones potentials, which gave good agreement with experimental data on PMMA thin films [27]. We note that, in order to follow a thermal spike with FENE samples, it was necessary to introduce variable time steps during the simulations. Such an approach is justified by the large initial excitation energy, which tends to make the interparticle distance in a chain $r$ close to $R_{0}$ in the beginning of the simulations, leading to divergences and integration errors. By making smaller time steps when the system was very hot, we could guarantee that no particle would move farther than a reference limit, keeping the simulation stable, while it evolves toward thermal equilibrium. 

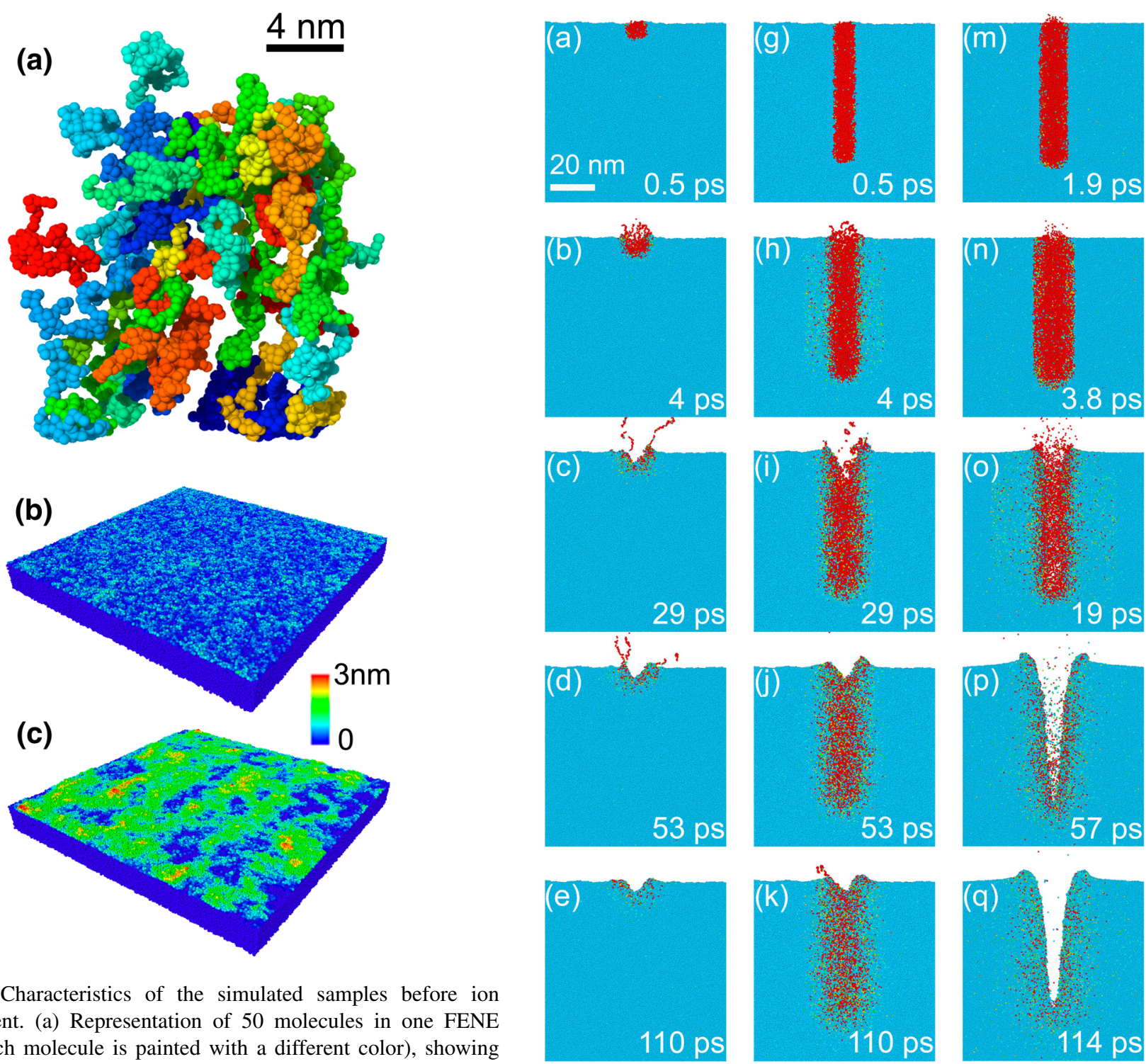

bombardment. (a) Representation of 50 molecules in one FENE sample (each molecule is painted with a different color), showing the presence of entanglements. (b) AFM-like images $\left(80 \times 80 \mathrm{~nm}^{2}\right)$ of pristine Lennard-Jones and (c) FENE films. The color code for the surface height is the same in (b) and (c). The FENE samples typically have a root mean square roughness of $\sim 0.25 \mathrm{~nm}$.

To model a thin film, the particles were labeled as belonging to the film or the substrate according to their initial depth within the sample (Fig. 1). The track was heated only within the film thickness (i.e., the film thickness is the excited track length). Thus, the substrate behaves as a material nonexcitable by the fast ions. This is a reasonable assumption for substrates made of silicon, where ion tracks are not observed. The simulated films have thicknesses $h$ in the range of 2-60 $\mathrm{nm}$.

The MD output files were analyzed using the open visualization tool (OVITO) [35]. Different cases were simulated three times or more, using different seeds in the velocity distribution function in order to evaluate statistical fluctuations. Also, the measurement of radiation-induced topological features were performed three times in different plane views in order to take geometrical variations also into account.
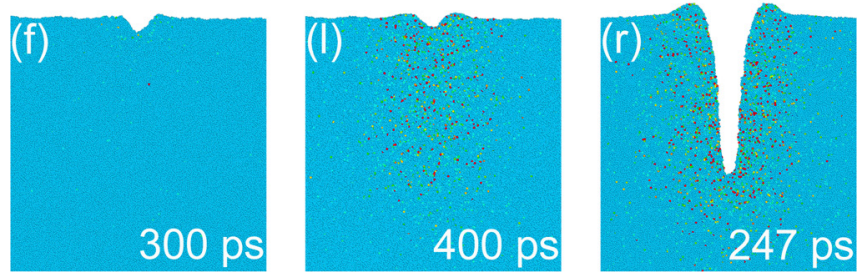

FIG. 3. Time evolution of a 5-nm FENE film [from (a) to (f)], a 60-nm FENE film [from (g) to (l)], and a 60-nm LJ film [from (m) to (r)] after the ion excitation. A color code for kinetic energy was employed, where red depicts particles with kinetic energies higher than the vaporization temperature and blue particles below melting conditions. For comparison purposes, melting and vaporization temperatures obtained for the LJ system were also kept in the FENE images.

\section{RESULTS}

Figure 3 shows the evolution of an ultrathin $(5 \mathrm{~nm})$ and a thick $(60 \mathrm{~nm})$ FENE film by looking at a slice in the middle of the simulation box. For comparison, similar results obtained 
for a 60-nm-thick LJ film are also shown. A color code for kinetic energy was employed, where red depicts particles with kinetic energies higher than the vaporization temperature and blue particles below melting conditions. For comparison purposes, the melting and vaporization temperatures obtained for the LJ system were also kept in the FENE images. The different stages of energy dissipation and transport, particle emission, crater formation, and relaxation can be seen in the different snapshots. There are important differences in the system evolution between the ultrathin and the thick film. For the ultrathin film, only the initially excited region of the track is melted and vaporized. There is a slight radial expansion of the vaporized zone [from 3 to $5 \mathrm{~nm}$, Figs. 3(a) and 3(b)], and after 29 ps, when energy dissipation by sputtering is seen, a molten region of $\sim 4 \mathrm{~nm}$ is formed. In this case, a rimless crater is formed and the film is cooled down very quickly. For the thick film, after about $4 \mathrm{ps}$, a vaporized phase coexists with a much larger molten region extending radially up to $\sim 16 \mathrm{~nm}$ (four times larger than in the 5-nm-thick film). The vaporized region expands also to a larger radius, reaching $\sim 7 \mathrm{~nm}$. At $29 \mathrm{ps}$, a hole with a depth of $\sim 20 \mathrm{~nm}$ is formed, but a subsequent flow of molecules into the cavity reduces substantially the final depth of the crater. It is very clear that the excited region and neighborhoods are kept hot for much longer times in the thicker film.

When comparing energy transport in FENE and LJ films of equal thickness, there are qualitative aspects that remain the same: First, there is a quick energy dissipation, which can be associated with a pressure pulse, followed by a slower diffusional dissipation during the cooling of the molten region. We computed the displacement of particles from their original positions in the beginning of the simulation, and several particles are seen to move coherently. During the simulation, this correlated movement changes into a Brownian-like movement, indicating that after the pressure pulse, energy is transferred as heat. Most particles in deep layers move radially, outward from the track core. Particles close to the surface have an upward momentum component, while those close to the bottom of the film tend to have a downward movement.

Despite such similarities, the presence of a chain structure impacts the system evolution, introducing several differences in the final crater shape observed for FENE and LJ thick films. The mobility of particles is severely restricted in the FENE system, where even very hot molecules may not escape the film. In contrast, hot-particle ejection is very pronounced in the LJ system. Additionally, in the LJ film, melt flow contributes substantially to the transport of mass towards the surface, resulting in a crater hole roughly the size of the entire film thickness and large rims. In FENE samples, however, melt flow acts to repopulate the cavity formed in the early stages of the track evolution, as can be seen from Figs. 3(i)-3(j). That is, the hot large molecules in the deep layers move a little upwards, before the systems cool sufficiently to freeze molecular motion. Thus, while melt flow diminishes the final crater depth in the FENE system, in the LJ films it acts as an important mechanism to enlarge the cavity size.

Figure 4 shows the top views and lateral slices of the final crater morphology at the end of a simulation of irradiated 20-nm-thick LJ and FENE films. FENE samples present much
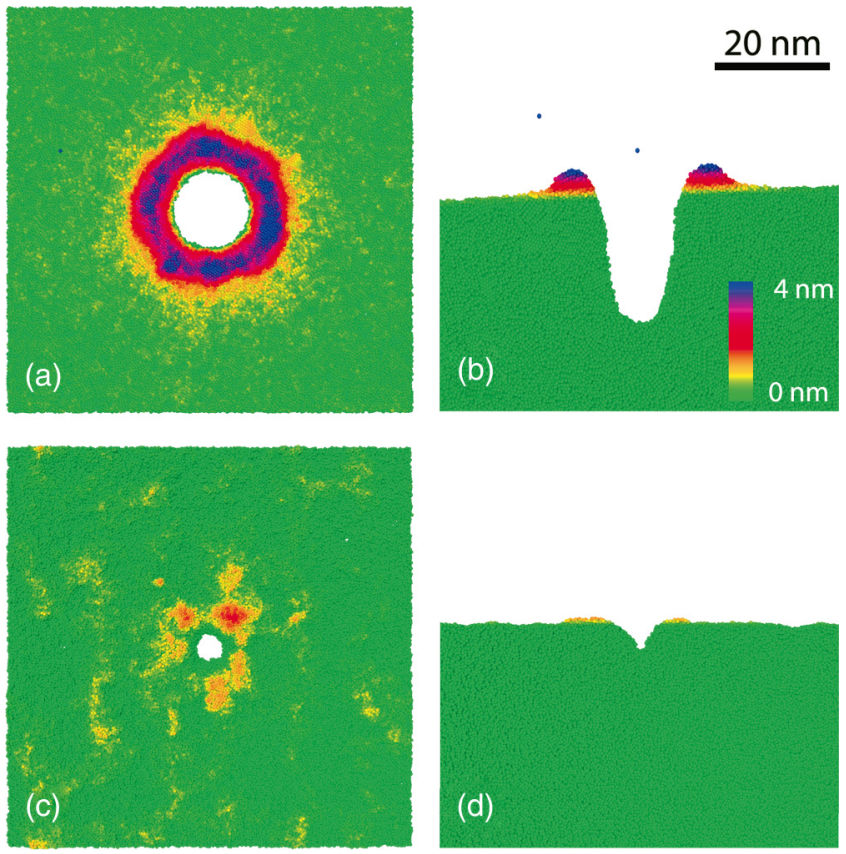

FIG. 4. Top and side views of (a), (b) Lennard-Jones and (c), (d) FENE films $(h=20 \mathrm{~nm})$ at the end of a simulation, color coded for surface height. In the FENE films, crater holes and rims are much smaller and less regular.

smaller and less uniform craters when compared with LJ samples (both the diameter and the depth of the crater are smaller). Crater rims are also small in FENE samples, being slightly larger than the initial roughness of the films, while in the LJ films they are very pronounced.

Particle ejection is also influenced by the presence of long chains. In the LJ sample, particles are emitted individually or as small clusters, and in the FENE system only entire molecules can be ejected, because bond breaking is artificially blocked. The decreased molecular mobility in the FENE samples results in much lower sputtering yields compared to LJ samples. For 60 -nm-thick films, the yield is $\sim 1400$ particles/ion in the LJ system, reducing to $\sim 800$ particles/ion for the FENE solid. In addition, changes in the chain conformation are clearly seen during the sputtering process in FENE solids. Many molecules that were in a coiled conformation in the beginning of the simulation adopt an extended conformation during ejection, and return to a compact shape after escaping the solid and cooling down. Thus, part of the excitation energy of the molecules is transferred to internal vibrational modes during the ejection process, which in a real material could lead to in-flight fragmentation. Similar changes in conformation during ejection were also seen in simulations of $\mathrm{keV}$ ions bombarding polymeric molecular solids [31,32].

We also evaluated the effect of film thickness in the crater size and sputtering yield. Figure 5 shows top view images of single ion impacts on films of different thicknesses, where the main qualitative differences can be appreciated. The trend found for the FENE films is similar to what has been recently observed for LJ films [27], with craters becoming strongly thickness dependent below a certain critical thickness that is different for craters and rims. Rim formation is much more 


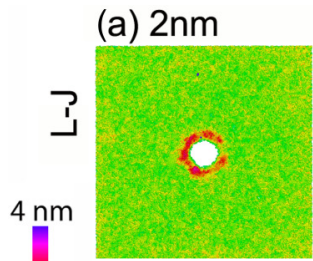

(b) $5 \mathrm{~nm}$

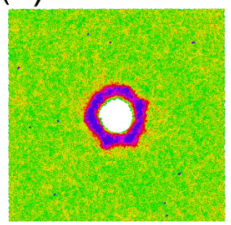

(f) $2 \mathrm{~nm}$

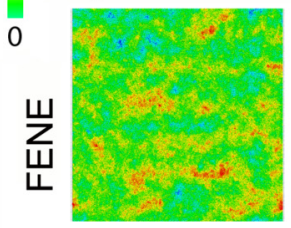

(g) $5 \mathrm{~nm}$

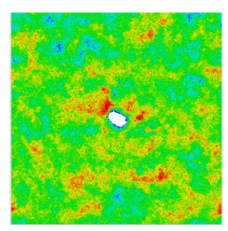

(c) $10 \mathrm{~nm}$

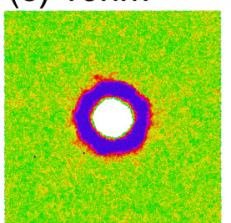

(h) $10 \mathrm{~nm}$

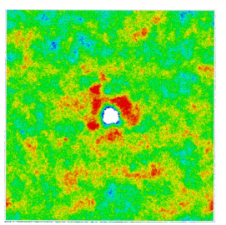

(d) $20 \mathrm{~nm}$

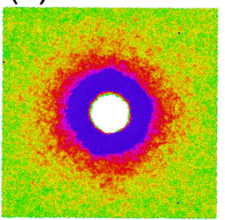

(i) $20 \mathrm{~nm}$

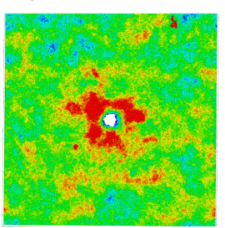

(e) $40 \mathrm{~nm}$

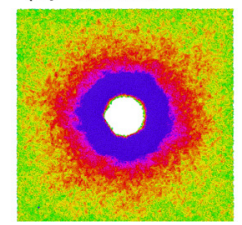

(j) $50 \mathrm{~nm}$

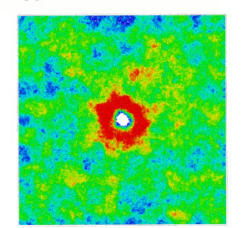

FIG. 5. Top view images (color coded for surface height) of (a)-(e) simulated LJ and (f)-(j) FENE films of different thicknesses (from 2 to $50 \mathrm{~nm}$ ) after the impact of a fast ion. The lateral size of the images is $80 \mathrm{~nm}$. Regions below the surface reference level $(z=0)$ appear white. At $h=2 \mathrm{~nm}$ no crater is formed in the FENE system.

sensitive to the reduction in the thickness of the film than the crater hole. Note that the ion impacts generate craters in LJ films for all thicknesses tested, but for the FENE films at $h=2 \mathrm{~nm}$ no crater is formed. This occurs when the track length is similar to the mean gyration radius $R_{g}$. For very short tracks, only a fraction of the particles in the chain is excited. Even for thicker films, many excited molecules in the simulation start to escape the solid, but recoil back to the surface, most probably because of interchain entanglement. As $R_{g}$ grows with chain length, the simulations indicate that the minimum thickness for crater formation (and intact molecular ejection) will increase with the molar mass of the polymer.

The mean crater depth $\left(Z_{\text {crater }}\right)$, crater diameter $\left(D_{\text {crater }}\right)$, and rim volume are displayed as a function of the film thickness in Fig. 6, allowing for a more detailed comparison between FENE and LJ results. In the FENE films, $Z_{\text {crater }}$ grows quickly with $h$ after the threshold at $h \sim 2 \mathrm{~nm}$ and already at $h \sim 10 \mathrm{~nm}$ reaches its maximum value. Beyond that, there is a slight decrease in crater depth until, at $h \sim 30 \mathrm{~nm}$, a plateau region, representing the bulk behavior, is reached. This plateau is clearly seen in the experimental data for $h>10 \mathrm{~nm}$. Thus, for the FENE system, the deepest craters are not found at the thickest films. This is related in part to the restricted mobility of the chains, and to the net number of particles moving into and out of the forming crater, which depends on the length of the excited track. Figure 7, which shows vectors of particle displacement from original positions for 5- and 35-nm-thick films, clearly illustrates this situation. In the thin film, while highly excited molecules in the track core leave the material, there is almost no melt flowing into the crater, because the heated track is very short. On the other hand, in the $35-\mathrm{nm}$ film there is a large number of heated particles from the deep regions of the excited track that cannot escape the film, but move into the cavity. Such an effect is not seen in the LJ films, because the melt, being very mobile for longer times, contributes to an increased crater depth at all tested thicknesses. Differently from the FENE, in the LJ films, $Z_{\text {crater }}$ increases steadily with $h$ in the entire range of investigated thicknesses. Even at $h=60 \mathrm{~nm}, Z_{\text {crater }}$ is far from reaching a plateau. Thus, while easy flow of the melt allows a large displacement of particles and enlarged craters in LJ thick films, the reduced mobility of the "viscous" melt in the FENE film acts to reduce the crater depth.

$D_{\text {crater }}$ also has a region at small $h$ where it is strongly thickness dependent, followed by a plateau region where a further increase in the track length produces no additional changes [Fig. 6(b)]. In this respect, FENE and LJ films present a similar qualitative behavior, although craters are much narrower in the FENE system and reach the plateau level at lower thicknesses $(\sim 10 \mathrm{~nm})$ compared to the LJ case $(\sim 20 \mathrm{~nm})$. Experiments indicate a critical thickness of $\sim 10 \mathrm{~nm}$ below which the crater diameter drops quickly [27], in good agreement with FENE results.

The volume of the crater rim as function of film thickness is displayed in Fig. 6(c). Again, FENE rims are smaller when compared to the LJ films for all thicknesses. For very thin films, the rim in the FENE system is very close to natural roughness of the surface. For both the FENE and LJ samples the rim volume grows with increasing thickness until a plateau value is reached. The plateau starts at similar film thicknesses: about $35 \mathrm{~nm}$ for FENE solids and $30 \mathrm{~nm}$ for the LJ case.

The sputtering yield $(Y)$ also decreases below a certain film thickness [Fig. 6(d)]. This is a result already seen in the early simulation work of Fenyö et al. [26]. The yields are expected to increase until the thickness of the film reaches values equivalent to the maximum depth of origin of sputtered particles in the bulk material $\left(Z_{Y}^{\infty}\right)$. Beyond that $h, Y$ grows slowly upon a further increase in $h$, until saturation is reached. The computed values of the maximum depth of origin of sputtered particles as a function of $h\left(Z_{Y}^{h}\right)$ are given in Fig. 8. We defined $Z_{Y}^{h}$ as the depth below which $95 \%$ of the ejected particles originate. For the LJ molecular solid, $Z_{Y}^{\infty} \sim 12 \mathrm{~nm}$, and the saturation yield is reached at about $h=30 \mathrm{~nm}$. In the FENE films, only intact molecules are ejected, and because of that, the yield grows in steps of 100 particles (one molecule). The sputtering yield saturation thickness is around $h=35 \mathrm{~nm}$ for the FENE films, but $Z_{Y}^{\infty} \sim 5.5 \mathrm{~nm}$. This is surprising, considering that the maximum crater depth is $Z_{\text {crater }} \sim 3 \mathrm{~nm}$ [Fig. 6(a)]. Thus, FENE simulations show sputtered particles coming from below the final crater depth, contrary to what is seen in the LJ film, where sputtered particles come always from depths above $Z_{\text {crater }}$ (Fig. 8). This is also in contrast 


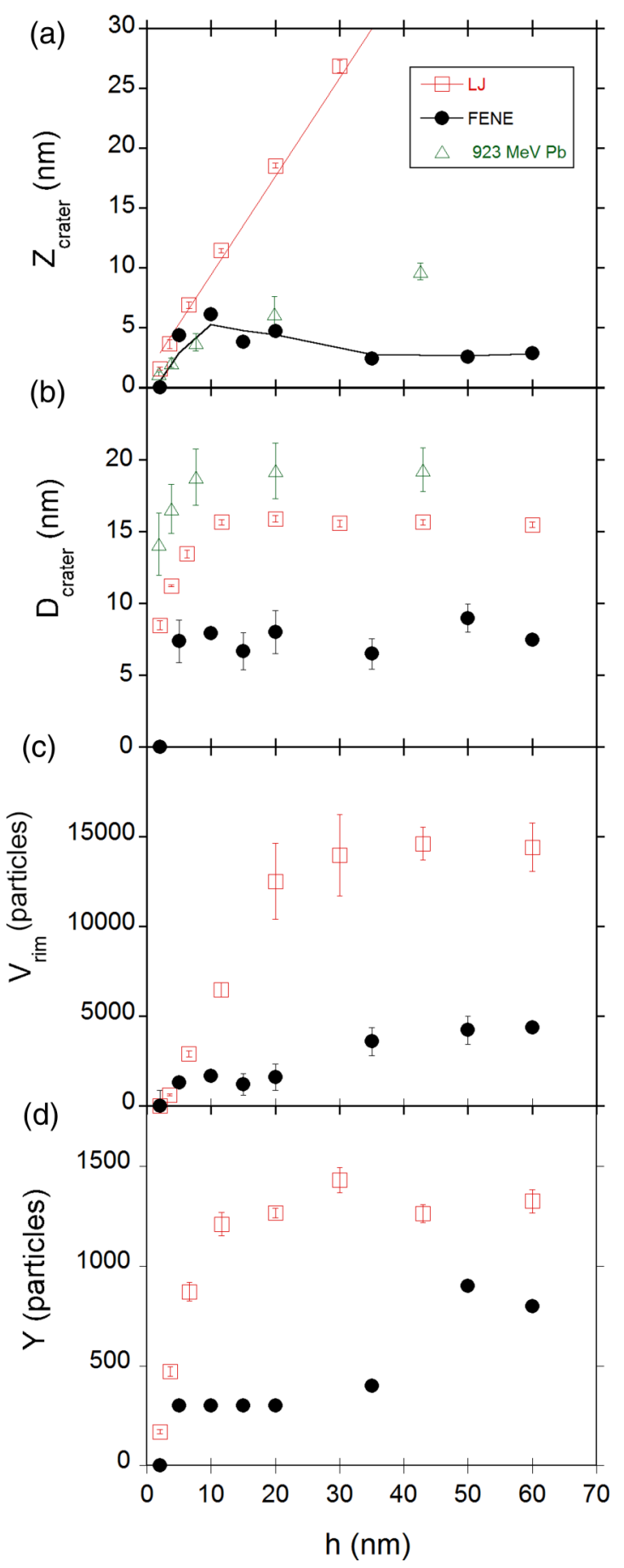

FIG. 6. (a) Crater depth, (b) crater diameter, (c) rim volume, and (d) sputtering yield for FENE samples as a function of film thickness. Data for LJ simulations and for experiments on PMMA films irradiated with $923 \mathrm{MeV} \mathrm{Pb} \mathrm{[27]} \mathrm{are} \mathrm{also} \mathrm{shown.} \mathrm{In} \mathrm{the} \mathrm{FENE}$ simulations, the measured crater diameters fluctuate more, in part due to the irregularity of the disposition of the molecules in the sample. Lines are guides to the eye.

to results obtained in simulations with $\mathrm{keV}$ ions, where the depth of origin of sputtering was found to be not greater than half of the crater depth [32]. Thus, in the FENE films, the displacement of molecules from deep layers contributes to the sputtering $Y$ and to diminishing the final crater volume.

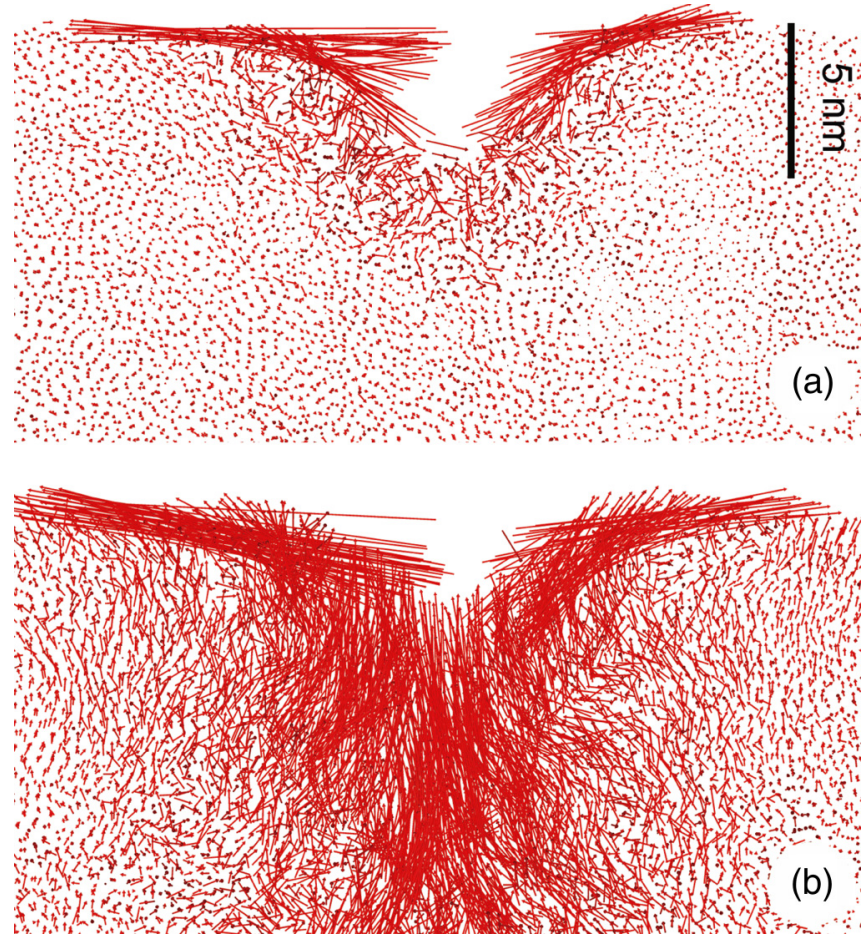

FIG. 7. Displacement vectors showing the displacement of particles from their original positions at the end of a simulation in a (a) 5-nm and (b) 35-nm FENE film. Note the important flow of particles into the crater hole for the thick film, which is negligible in the thinner film.

We finally note that, even for very thin films, the depth of origin of sputtered particles never reaches the entire film thickness and is always approximately below $h / 2$ (dashed line in Fig. 8). In the FENE samples, both the depth of origin and the crater depth are always smaller than $h / 2$, similarly to what was seen in the experiments with polymer films [27].

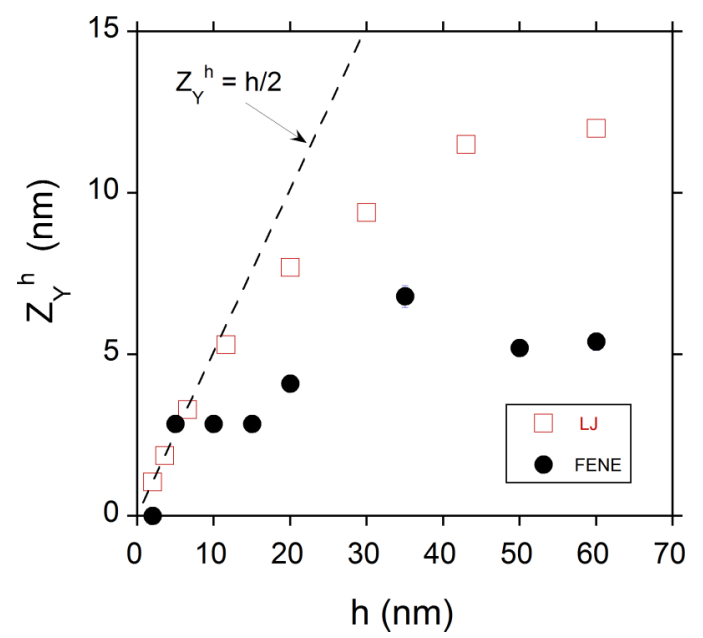

FIG. 8. Maximum sputtering depth of origin $Z_{Y}^{h}$ as a function of film thickness for FENE and LJ samples. The curve for $Z_{Y}^{h}=h / 2$ is also added as a dashed line. 


\section{CONCLUSIONS}

We presented simulations of surface effects produced by a thermal spike on polymerlike films due to the impact of a fast heavy ion, using a coarse-grained approach with the FENE potential. The evolution of the excited track, sputtering, and the final surface topology at the impact site were followed as a function of film thickness, and compared to results obtained previously with simulations using the Lennard-Jones (LJ) potential and experimental data with polymer thin films. Crater sizes and sputtering yields are reduced in the FENE systems, compared to LJ films for all thicknesses tested, and a threshold thickness for molecular ejection appears in FENE simulations. This is attributed to the additional restrictions to mass transport out of the excited track region imposed by interchain interactions (entanglements) and by the low mobility of the high molecular weight molten phase induced by the spike.

When comparing the simulation results to the experimental data in real polymers, the quantitative agreement is, in general, limited, because both potentials are far from representing a real macromolecule. Even though the crater features in FENE solids are smaller than what is seen experimentally, FENE simulations better match the morphology of the craters seen in polymers and can achieve better agreement with experimental rim volumes and crater depths than LJ simulations, due to added bonding along the chains. However, excitation details (track radius and temperature) were previously optimized to reproduce the crater diameter using the LJ potential [27], and additional optimization would be required to improve agreement with FENE simulations. Moreover, having an inactive bond-breaking channel, the FENE construct overestimates the system resistance to the ion impact. Fragmentation is expected in the inner part of the ion track in organic materials [36], and further work is now being conducted with more complex potentials capable of dealing with bond breaking and chemical reactions. This will also allow us to simulate the mass spectra of intact molecules and different fragments to compare to experiments of MeV-SIMS of large organic molecules.

\section{ACKNOWLEDGMENTS}

This work has been partially funded by CNPq and CAPES (Brazil). R.G. acknowledges support from Fondo Nacional de Investigaciones Científicas y Tecnológicas (FONDECYT, Chile) under Grant No. 3140526 and Financiamiento Basal para Centros Científicos y Tecnológicos de Excelencia FB0807 (CEDENNA). E.M.B. acknowledges support from PICT2014-0696 (ANPCyT), a SeCTyP-UN Cuyo grant. This work is part of the IAEA Coordinated Research Project on Development of Molecular Concentration Mapping Techniques using MeV focused ion beams, CRP F11019 (Contracts No. 18352/R0 and No. 18686).
[1] R. Helborg, H. Whitlow, and Y. Zhang, Ion Beam in Nanoscience and Technology (Springer, Berlin, 2009).

[2] R. L. Fleischer, P. Price, and R. M. Walker, Nuclear Tracks in Solids (University of California Press, Berkeley, 1975).

[3] J. R. Tesmer and M. Nastasi, Handbook of Modern Ion Beam Material Analysis (Materials Research Society, Pittsburgh, 1995).

[4] T. Kanai, M. Endo, S. Minohara, N. Miyahara, H. Koyama-Ito, H. Tomura, N. Matsufuji, Y. Futami, A. Fukumura, T. Hiraoka, Y. Furusawa, K. Ando, M. Suzuki, F. Soga, and K. Kawachi, Int. J. Radiat. Oncol. Biol. Phys. 44, 201 (1999).

[5] A. Meftah, F. Brisard, J. M. Costantini, E. Dooryhee, M. HageAli, M. Hervieu, J. P. Stoquert, F. Studer, and M. Toulemonde, Phys. Rev. B 49, 12457 (1994).

[6] R. M. Papaléo, in Fundamentals of Ion-Irradiated Polymers, edited by D. Fink (Springer, Berlin, 2004).

[7] B. U. R. Sundqvist and R. D. Macfarlane, Mass Spectrom. Rev. 4, 421 (1985).

[8] R. Neumann, Nucl. Instrum. Methods Phys. Res. B 151, 42 (1999).

[9] C. Jeynes, R. P. Webb, and A. Lohstroh, Rev. Accel. Sci. Technol. 4, 41 (2011).

[10] Y. Nakata, Y. Honda, S. Ninomiya, T. Seki, T. Aoki, and J. Matsuo, Appl. Surf. Sci. 255, 1591 (2008).

[11] J. D. Watrous, T. Alexandrov, and P. C. Dorrestein, J. Mass Spectrom. 46, 209 (2011).

[12] L. A. McDonnell and R. M. A. Heeren, Mass Spectrom. Rev. 26, 606 (2007).
[13] W. Ens, B. U. R. Sundqvist, P. Hakansson, A. Hedin, and G. Jonsson, Phys. Rev. B 39, 763 (1989).

[14] M. Toulemonde, W. Assmann, C. Trautmann, and F. Grüner, Phys. Rev. Lett. 88, 057602 (2002).

[15] O. Osmani, N. Medvedev, M. Schleberger, and B. Rethfeld, Phys. Rev. B 84, 214105 (2011).

[16] H. M. Urbassek and K. T. Waldeer, Phys. Rev. Lett. 67, 105 (1991).

[17] E. M. Bringa, R. E. Johnson, and M. Jakas, Phys. Rev. B 60, 15107 (1999).

[18] E. M. Bringa and R. E. Johnson, Nucl. Instrum. Methods Phys. Res. B 193, 365 (2002).

[19] E. M. Bringa, R. E. Johnson, and R. M. Papaléo, Phys. Rev. B 65, 094113 (2002).

[20] K. Nordlund and F. Djurabekova, J. Comput. Electron. 13, 122 (2014).

[21] R. Smith, M. Harrison, and R. Webb, Thin Solid Films 343, 602 (1999).

[22] J. D. Mody and R. P. Webb, Surf. Interface Anal. 43, 92 (2011).

[23] K. Nordlund, J. Peltola, J. Nord, J. Keinonen, and R. S. Averback, J. Appl. Phys. 90, 1710 (2001).

[24] E. M. Bringa and R. E. Johnson, Nucl. Instrum. Methods Phys. Res. B 143, 513 (1998).

[25] H. M. Urbassek, H. Kafemann, and R. E. Johnson, Phys. Rev. B 49, 786 (1994).

[26] D. Fenyö and R. E. Johnson, Phys. Rev. B 46, 5090 (1992). 
[27] R. M. Papaléo, R. Thomaz, L. I. Gutierres, V. M. de Menezes, D. Severin, C. Trautmann, D. Tramontina, E. M. Bringa, and P. L. Grande, Phys. Rev. Lett. 114, 118302 (2015).

[28] K. Kremer and G. S. Grest, J. Chem. Phys. 92, 5057 (1990).

[29] M. Bulacu and E. Van Der Giessen, J. Chem. Phys. 123, 114901 (2005).

[30] B. Z. Dlugogorski, M. Grmela, and P. J. Carreau, Rheol. Acta 34, 384 (1995).

[31] A. Delcorte, O. A. Restrepo, K. Hamraoui, and B. Czerwinski, Surf. Interface Anal. 46, 46 (2014).
[32] A. Delcorte and B. J. Garrison, J. Phys. Chem. C 111, 15312 (2007).

[33] S. Plimpton, J. Comput. Phys. 117, 1 (1995).

[34] G. Szenes, K. Havancsák, V. Skuratov, P. Hanák, L. Zsoldos, and T. Ungár, Nucl. Instrum Methods Phys. Res. B 166, 933 (2000).

[35] A. Stukowski, Model. Simul. Mater. Sci. Eng. 18, 015012 (2009).

[36] R. M. Papaléo, P. Demirev, J. Eriksson, P. Håkansson, B. U. R. Sundqvist, and R. E. Johnson, Phys. Rev. Lett. 77, 667 (1996). 